\title{
Guest editorial: special issue on the Real-Time Systems Symposium 2017
}

\author{
Isabelle Puaut ${ }^{1}$
}

Published online: 11 March 2019

(c) Springer Science+Business Media, LLC, part of Springer Nature 2019

Real-time computing plays a crucial role in our society since an increasing number of systems relies on processor control. Examples of applications that require realtime operations include air traffic control, automotive applications in particular in autonomous vehicles, robotics and medical devices. Embedded computers are integral parts of these systems that have to keep up with the temporal dynamics of their physical environment. As a consequence, many of them feature real-time requirements, i.e. have to react within timing constraints while still producing correct results.

IEEE Real-Time Systems Symposium (RTSS) is the premier conference in the area of real time systems, presenting innovations in the field with respect to theory and practice. RTSS provides a forum for the presentation of high-quality, original research covering all aspects of real-time systems theory, design, analysis, implementation, evaluation, and experiences. RTSS 2017 continues the trend of making RTSS an expansive and inclusive symposium, looking to embrace new and emerging areas of real-time systems research.

In 2017, RTSS was celebrated in beautiful Paris, France, and reached its 38th edition, presenting a very interesting program that included excellent scientific papers carefully selected by the program committee, a brief presentation session, four satellite workshops and an open demo session. A selection of the best papers presented at the conference was made, and their authors were invited to submit extended journal versions of their papers to this special issue of the Real-Time Systems Journal. These journal papers were reviewed according to the rigorous standards of the Real-Time Systems Journal.

The first work in this special issue, by Zhen Dong and Cong Liu, addresses an important challenge in multiprocessor scheduling: the scheduling of gang task systems under earliest deadline first, where a gang application's threads need to be concurrently scheduled on distinct processors. The authors introduce a novel approach combining new lag-based reasoning and executing/non-executing gang interval analysis technique, which is able to characterize the parallelism- induced idleness, as a key challenge

\footnotetext{
$凶 \quad$ Isabelle Puaut puaut@irisa.fr

1 Univ Rennes, Inria, CNRS, IRISA, Rennes, Italy
} 
of analyzing gang task schedules. This approach yields the first utilization-based test for hard real-time gang task systems.

The second paper, by Yecheng Zhao and Haibo Zeng considers the problem of design optimization for real- time systems scheduled with fixed priority, where task priority assignment is part of the decision variables, and the timing constraints and/or objective function linearly depend on the exact value of task response times. The paper proposes an efficient optimization framework that is three orders of magnitude (1000 times) faster than Integer Linear Programming (ILP) while providing solutions with the same quality.

We would like to thank all the authors who have submitted papers to the Special Section, we are truly grateful for the efforts and patience of the reviewers whose expertise guarantees the outstanding quality of the journal issue.

Publisher's Note Springer Nature remains neutral with regard to jurisdictional claims in published maps and institutional affiliations.

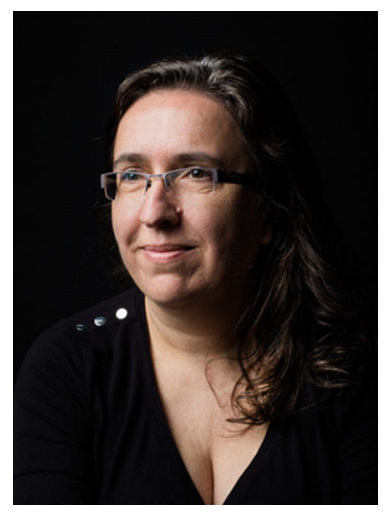

Isabelle Puaut received a $\mathrm{PhD}$ degree in computer science from the University of Rennes in 1993. After 10 years as an assistant professor at INSA (Institut National des Sciences Appliquées, engineering school), she is since 2004 a professor in computer science at the university of Rennes. Her research interests include real-time systems, computer architecture, programming languages, and operating systems. Her current research focuses on timing analysis of real-time software (worst case execution times estimation), more precisely on the impact of hardware on worst-case performance. She has published more than 130 papers in journal publications or peer-reviewed conference papers. 